\title{
On-Line Automatic Detection of Driver Drowsiness Using a Single Electroencephalographic Channel
}

\author{
Antoine Picot, Sylvie Charbonnier and Alice Caplier
}

\begin{abstract}
In this paper, an on-line drowsiness detection algorithm using a single electroencephalographic (EEG) channel is presented. This algorithm is based on a means comparison test to detect changes of the alpha relative power $([8-12] \mathrm{Hz}$ band). The main advantage of the method proposed is that the detection threshold is completely independent of drivers and does not need to be tuned for each person. This algorithm, which works on-line, has been tested on a huge dataset representing 60 hours of driving and give good results with nearly $85 \%$ of good detections and $20 \%$ of false alarms.
\end{abstract}

\section{INTRODUCTION}

Drowsiness is the transition state between awakening and sleep during which a decrease of the vigilance, i.e. the capacity of keeping oneself attention on a task, is generally observed. This can be a serious problem for tasks that need a sustained attention, such as driving. According to a report of the American National Highway Safety Traffic Administration (NHSTA) [1], driver drowsiness is annually responsible for about 56,000 crashes which is the reason why more and more researches are made to build automatic detectors of this dangerous state.

This paper proposes a drowsiness detection algorithm using a single EEG channel based on a means comparison test. In a first section, a brief state of the art on automatic detection using physiological information is made. Then, the method is presented in section III. Finally, the results obtained on a significant database of EEG recordings from drowsy drivers are shown and discussed.

\section{STATE OF THE ART}

Electroencephalography measures the electrical activity of the brain from electrodes placed on the scalp. EEG is described in term of rhythmic activity and transients. The rhythmic activity is divided into frequency bands: delta $(\delta)$ activity $([0.5-4] \mathrm{Hz})$, theta $(\theta)$ activity $([4-8] \mathrm{Hz})$, alpha $(\alpha)$ activity $([8-12] \mathrm{Hz})$, beta $(\beta)$ activity $([12-26] \mathrm{Hz})$ and gamma $(\gamma)$ activity (over $26 \mathrm{~Hz}$ ). Most of the time, only the range [1-20] Hz is used because activity below or above this range is likely to be artifactual (under standard clinical recording techniques).

Drowsiness is characterized by an increase of $\alpha$ and $\theta$ activities, predominantly in the posterior region of the brain, and a slowdown of blinks and eye movements [3]. Different scales of drowsiness classification exist but there are no standardized rules to differentiate the levels of drowsiness (as

A.Picot, S.Charbonnier and A.Caplier are with Gipsa-lab, 961 rue de la Houille Blanche, Domaine Universitaire - BP 46, 38402 Saint Martin d'Heres Cedex, France name. surnamedgipsa-lab. inpg. fr
TABLE I

OSS CRITERIA

\begin{tabular}{|c|c|c|}
\hline $\begin{array}{c}\text { Objective sleepiness } \\
\text { score }\end{array}$ & $\begin{array}{c}\alpha \text { and } \theta \\
\text { cumulative duration }\end{array}$ & $\begin{array}{c}\text { Blinks and eye } \\
\text { movements }\end{array}$ \\
\hline $\mathbf{0}$ & negligible & Normal \\
\hline $\mathbf{1}$ & less than $5 \mathrm{~s}$ & Normal \\
\hline $\mathbf{2}$ & $\begin{array}{c}\text { less than } 5 \mathrm{~s} \\
\text { or } \\
\text { less than } 10 \mathrm{~s}\end{array}$ & Slow \\
\hline $\mathbf{3}$ & $\begin{array}{c}\text { less than 10s } \\
\text { or } \\
\text { more than } 10 \mathrm{~s}\end{array}$ & Slow \\
\hline $\mathbf{4}$ & more than 10s & Normal \\
\hline
\end{tabular}

the Rechtschaffen and Kales rules [2] for the study of sleep). This may be due to the quite recent interest on drowsiness compared to the sleep analysis.

There are two kinds of scales: subjective sleepiness scales like the Karolinska Sleepiness Scale (KSS) [4] which allow drivers to directly evaluate their own drowsiness and Objective Sleepiness Scales (OSS) which is used by expert doctors to evaluate drivers drowsiness after driving. The OSS used in this study is a five-level scale from 0 (awake) to 4 (very drowsy) developped by Muzet [3]. Decisions are made every $20 s$ and depend on the length of $\alpha$ and $\theta$ bursts as well as on the speed of eyes movements and blinks. The different criteria are presented in table I.

Most of drivers' drowsiness automatic detection methods are vehicle-oriented, studying the vehicle behavior to detect abnormal doings [5], or face-oriented, using video to detect blinks and head movements [6], [7]. Physiological studies on drowsiness are based on EEG analysis and only a few are about real-time monitoring systems. The EEG power spectrum is used to evaluate the activity in each band. De Waard [8] suggests to monitor the ratio $\frac{\alpha+\theta}{\beta}$ to estimate the drowsiness level. The EEG power spectrum can also be used to train neural networks [9], [10]. The point is that methods based on neural networks need a huge expertized database to train the network. More recent studies are based on Independent Component Analysis (ICA) [11], [12]. ICA methods need to have a large number of EEG channels but allow the spatial shifting of the different bands activities to be tracked which bring new information in addition to the EEG power spectrum.

The method described in the following section uses the EEG power spectrum to detect bursts in the EEG activity and to estimate if the driver is drowsy or not. 


\section{DROWSINESS DETECTION METHOD}

The principle of the detection method is shown on Fig. 1 . First the EEG power spectrum is computed using a Short

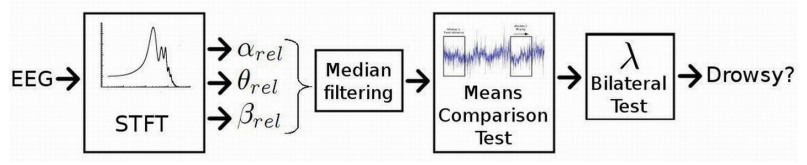

Fig. 1. Drowsiness detection method

Time Fourier Transform (STFT) to calculate the relative energy into the different EEG bands every second. Then, the relative energy is median filtered using a sliding window to reject abnormal values. A Means Comparison Test (MCT) is at last computed to compare the energy to a reference level, learnt at the beginning of the recording. As the MCT is normalized, experiment will help to determine a common threshold of detection.

\section{A. EEG Power Spectrum}

The EEG power spectrum is computed using a STFT. The power spectrum is computed on the two past seconds using Welch's periodigram method, with an overlapping window of $1 s$ [13]. It is computed every second. Then, the relative power of each band is calculated. For example the $\alpha$ relative power is calculated as following:

$$
\alpha_{\text {relative power }}=\frac{\alpha_{\text {power }}}{E E G_{\text {power }}},
$$

the relative powers in the bands $\theta$ and $\beta$ are calculated by similarity. The relative power in the bands $\alpha, \theta$ and $\beta$ will be respectively named $\alpha_{r e l}, \theta_{\text {rel }}$ and $\beta_{\text {rel }}$.

\section{B. Median filtering}

Median filtering is used to smooth the $\alpha_{\text {rel. }}, \theta_{\text {rel. }}$ and $\beta_{\text {rel }}$ signals and to reject abnormal values. The median is the value separating the higher half of a population from the lower half. Here, the median of the relative powers is calculated every second, before performing MCT, using a sliding window of $10 \mathrm{~s}$.

\section{Means Comparison Test}

The method of MCT is inspired by [14] and is applied on the relative powers in the $\alpha, \theta$ and $\beta$ bands. A moving window is compared to a fixed reference window as shown on fig. 2. The classical MCT has quite restrictive conditions due to the fact that the theoretical variances are unknown. Let us consider two independent populations of length $n_{1}$ and $n_{2}$, whose means are $\bar{x}_{1}$ and $\bar{x}_{2}$ and whose variances are $s_{1}^{2}$ and $s_{2}^{2}$. Then, the variable:

$$
t=\frac{\bar{x}_{1}-\bar{x}_{2}}{\sqrt{\frac{n_{1} s_{1}^{2}+n_{2} s_{2}^{2}}{n_{1}+n_{2}-2}\left(\frac{1}{n_{1}}+\frac{1}{n_{2}}\right)}}
$$

follows a $n_{1}+n_{2}-2$ liberty degrees Student law. The equality of the two means can be tested by a bilateral test with a confidence threshold $\lambda:-t_{1-\lambda / 2}<t<t_{1-\lambda / 2}$.

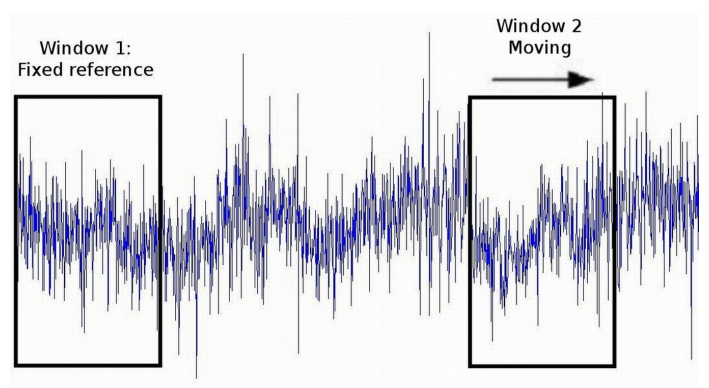

Fig. 2. Illustration of the windows for the MCT

If the two populations have the same length $n$ (i.e. $n_{1}=$ $n_{2}=n$ ) and their theoretical variances are equal, (1) can be formulated as:

$$
t=\frac{\bar{x}_{1}-\bar{x}_{2}}{\sqrt{\frac{s_{1}^{2}+s_{2}^{2}}{n-1}}}
$$

So, the variable $t$ follows a $n-1$ liberty degrees Student law.

Moreover, if the populations are large enough, i.e. if $n_{1}$ and $n_{2}$ are equal or greater than 20, the test is performed with the variable:

$$
u=\frac{\bar{x}_{1}-\bar{x}_{2}}{\sqrt{\frac{s_{1}^{2}}{n_{1}}+\frac{s_{2}^{2}}{n_{2}}}}
$$

which then follows a centered reduced normal law. The means equality is then tested by a bilateral test with a confidence threshold $\lambda:-u_{1-\lambda / 2}<u<u_{1-\lambda / 2}$. Furthermore, the theoretical variances equality is not longer necessary.

Here, the test is computed on the relative power signals calculated every second. The length of the fixed reference window is $n_{1}=60 s$ and the one of the moving window is $30 s$ with $29 s$ overlap. The threshold $\lambda$ fixes the percentage of false alarms expected. The higher the threshold, the lesser the percentage of false alarms. In this study, $\lambda$ is empirically chosen and discussed in section IV.

\section{Method relevance}

The reference is calculated on a fixed window chosen at the beginning of the signal, supposing that the driver is completely awake when he starts driving. So, the mean calculated on the moving window is compared to a wakefulness reference. If the bilateral test is higher than the threshold, the driver is then considered as drowsy.

A big problem with EEG analysis is the occurrence of artifacts. Artifacts are perturbations of the EEG signal due to patient movements such as ocular movements or measurement devices such as electrode disconnections. Highamplitude artifacts pollute the EEG signals and give isolated high abnormal values on the whole EEG band of the power spectrum. The median filter is used to reject this values to avoid false detection.

The point with detecting $\alpha$ or $\theta$ bursts in EEG signal is the difficulty to define a common threshold for all drivers because of the large inter-individuals differences [16]. Here, 
the level of $\alpha_{\text {rel }}$ and $\theta_{\text {rel }}$ power in the awake state is learned on each driver from the reference window. Moreover, the output of MCT is a variable following a centered reduced normal law. So, the threshold used in the bilateral test has statistical meaning and is the same for all drivers.

At last, the whole algorithm can be applied on-line. However, the sliding window of $10 \mathrm{~s}$ used for median filtering induces a delay of $5 \mathrm{~s}$ and the sliding window of $30 \mathrm{~s}$ used for the MCT induces $15 \mathrm{~s}$. So, the decision provided by the algorithm is delayed by $20 s$ from the signals recorded. This delay in the decision will be taken into account when comparing the results to the expert's decisions.

\section{RESULTS AND DISCUSSION}

\section{A. Database}

The database used for the evaluation of the method is composed of 40 recordings from twenty subjects. Subjects were tested on the moving base driving simulator PAVCAS ("Poste d'Analyse de la Vigilance en Conduite Automobile Simulée"). Each subject was recorded while driving for 90 minutes, a first time perfectly rested and a second time suffering from sleep deprivation (the subject had slept for 4 hours only). The data base is thus composed of 60 hours. Each recording includes four EEG channels (left frontal (F3), central (C3), parietal (P3) and occipital (O1)), one EOG channel and a video of the driver's face. Objective sleepiness was evaluated on each recording by an expert doctor using the scale described in section II. Data acquisition was performed at $250 \mathrm{~Hz}$ by the CEPA (Centre d'Études de Physiologie Appliquée), Strasbourg, FR.

\section{B. Technical validation}

The method proposed in this paper provides a binary decision [awake; drowsy] while the database is expertized using five levels. Moreover, the expert classified non overlapping intervals of $20 \mathrm{~s}$ (epochs) while the automatic system made a decision every second. To compare the results obtained to the expert's decision, the following validation technique was used. The five expert decision levels were converted into a binary decision by considering as drowsy any decision superior or equal to 1 in the expert's scale. Furthermore, each $20 s$ epoch classified by the expert was directly compared to the system decision: if during the $20 \mathrm{~s}$ interval, the system classified at least 1s as "drowsy", then the decision for the epoch was "drowsy". Else it was "awake".

Epochs were then compared one by one and classified according to the contingency table II. The true positive rate $\left(T P_{\text {rate }}\right)$ or detection rate is the ratio between the number of true "drowsy" automatic decisions and the number of "drowsy" expert decisions. The false positive rate $\left(F P_{\text {rate }}\right)$ ) is the ratio between the number of false "drowsy" automatic decisions and the number of "awake" expert decisions. They are calculated according (4) and (5).

$$
\begin{aligned}
& T P_{\text {rate }}=\frac{T P}{T P+F N} \\
& F P_{\text {rate }}=\frac{F P}{F P+T N}
\end{aligned}
$$

TABLE II

\begin{tabular}{|c|c|c|c|}
\hline & & Exper & lecision \\
\hline & & awake & drowsy \\
\hline Automatic & awake & $\begin{array}{c}\text { True Negative } \\
\text { (TN) }\end{array}$ & $\begin{array}{c}\text { False Negative } \\
\text { (FN) }\end{array}$ \\
\hline decision & drowsy & $\begin{array}{c}\text { False Positive } \\
\text { (FP) }\end{array}$ & $\begin{array}{c}\text { True Positive } \\
\text { (TP) }\end{array}$ \\
\hline
\end{tabular}

CONTINGENCY TABLE

The results are displayed as Receiver Operating Characteristic (ROC) curves [15], plotting $T P_{\text {rate }}$ in function of $F P_{\text {rate }}$. The purpose is to have the highest $T P_{\text {rate }}$ with the lowest $F P_{\text {rate }}$.

\section{Results and discussion}

The drowsiness detection algorithm was applied on the whole database, with a decision threshold $\lambda$ (defined in section III.C) varying from 1.5 to 5 , on each of the 4 EEG channels. The results obtained when the MCT is applied on the alpha relative power is presented in Fig. 3. The "star" markers correspond to the P3 channel, the "circle" markers to the F3 channel, the "square" markers to the C3 channel and the "triangle" markers to the $\mathrm{O} 1$ channel. The head at the bottom on the right reminds the position of each channel. For each channel, the results represented with the marker the further on the right corresponds to the smallest $\lambda$ and with the marker the further on the left to the biggest $\lambda$. It is coherent: increasing $\lambda$ diminishes the $F P_{\text {rate }}$ while decreasing the $T P_{\text {rate. }}$ It is obvious from Fig. 3 that the results are much

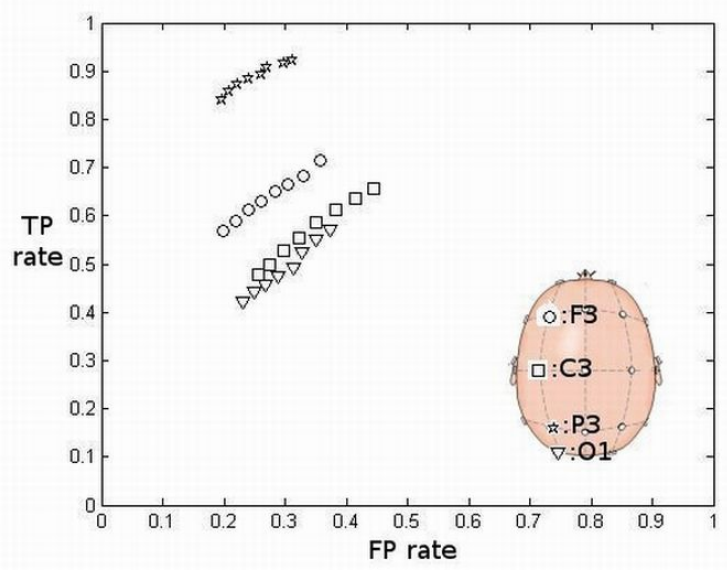

Fig. 3. Results of the drowsiness detection algorithm on each EEG channel

better when the P3 posterior channel is used, which is in concordance with results from the literature: drowsiness is caracterized by an increase of $\alpha$ activity predominately in the posterior region of the brain.

Fig. 4 compares the results obtained using EEG recorded from channel P3 with MCT applied to:

- $\alpha_{\text {rel }}$ ("star" markers) 
- $\alpha_{\text {rel }} \mid \theta_{\text {rel }}$ ("square" markers)

- $\frac{\alpha+\theta}{\beta}$ ("circle" markers) suggested in [8]

- $\alpha_{\text {rel }}$ ("triangle" markers) signal without the median filter

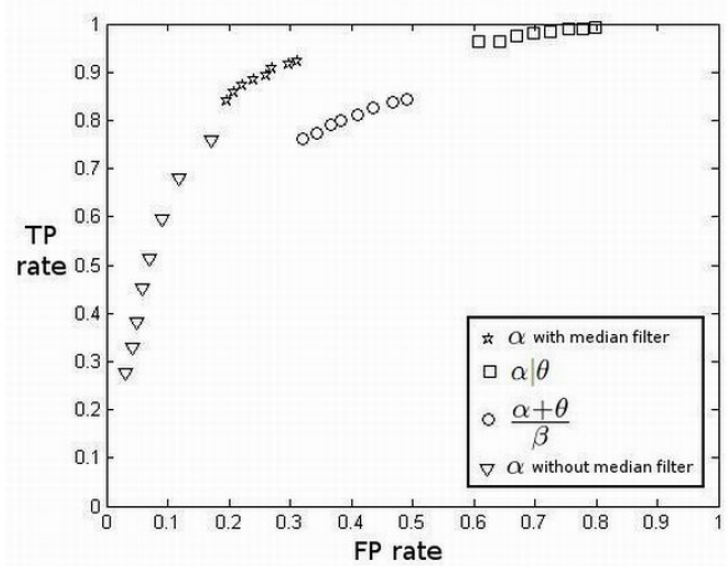

Fig. 4. Comparison between different criteria of drowsiness detection

The best results are obtained with the drowsiness detection algorithm applied on the $\alpha_{\text {rel }}$ signal. The optimal results are $T P_{\text {rate }}=84,16 \%$ and $F P_{\text {rate }}=19,59 \%$ with a threshold $\lambda=5$. However, decreasing the threshold $\lambda$ from 5 to 3 does not degrade the performances $\left(T P_{\text {rate }}=89,28 \%\right.$ and $F P_{\text {rate }}=25,99 \%$ with $\lambda=3$ ), which proves that the method is not sensitive to the threshold value. The results obtained with the $\alpha_{r e l}$ signal without the median filter are good for $\lambda=1.5$ but they quickly decrease when increasing $\lambda$. They are very sensitive to the threshold value. So, the median filter improves the results and make the method less sensitive to the threshold value. Moreover, the algorithm was tested with the same threshold on data recorded from 20 different patients, which shows that the method can be applied on any patient without adapting the tuning parameter.

Finally, the results obtained with $\alpha_{\text {rel }} \mid \theta_{\text {rel }}$ show that the $\theta_{\text {rel }}$ is not relevant to detect drowsiness since the number of false positive increase tremendously when this information is addes. In the same way, the $\frac{\alpha+\theta}{\beta}$ ratio gives correct results but less good than the results obtained with the only $\alpha_{\text {rel }}$ information.

At last, this detection algorithm can operates on-line in real time, giving decisions every second. The decision delay of $20 \mathrm{~s}$ is not important when compared to the physician's decision made every $20 \mathrm{~s}$.

\section{CONCLUSIONS AND FUTURE WORKS}

An universal algorithm for the on-line automatic detection of drivers drowsiness has been presented here. This algorithm is based on a means comparison test applied on the EEG relative power calculated in the alpha band. This algorithm can operate on-line with a short delay and is tuned by a threshold whose value is independent of the drivers. Performances were shown not to be sensitive to significant changes in the tuning parameter. The results obtained are $84,2 \%$ of good detection and $19,6 \%$ of false detection using only one EEG channel.

The next step of this work is to add an "eye blinks and yawn" detection system thanks to a high frame rate camera and to merge the decisions to obtain a highly reliable automatic drowsiness detector.

\section{ACKNOWLEDGMENTS}

The authors are grateful to the Centre d'Études de Physiologie Appliquée (CEPA) in Strasbourg (FR) for providing the data and their help, as well as the Laboratoire d'Automatique, de Mécanique, et d'Informatique industrielles et Humaines (LAMIH) in Valenciennes (FR).

\section{REFERENCES}

[1] D. Royal, National Survey of Distracted and Drowsy Driving Attitudes and Behavior, DOT HS 809 566, 2002, pp 41-53.

[2] A.Rechtschaffen and A.Kales, A Manual of Standardized Terminology, Techniques and Scoring System for Sleep Stages of Human Subject, US Government Printing Office, National Institute of Health Publication, Washington DC, 1968.

[3] A. Muzet, T. Pébayle, J. Langrognet \& S. Otmani, AWAKE Pilot study no.2: Testing steering grip sensor measures, IST-2000-28062, 2003.

[4] T. Akerstedt and M. Gillberg, "Subjective and objective sleepiness in the active individual", International Journal of Neuroscience,vol. 52, pp. 29-37, 1990.

[5] A. Kircher, M. Uddman and J. Sandin, "Vehicle control and Drowsiness", Swedish National Road and Transport Research Institute, 2002.

[6] Q. Ji, Z. Zhu and P. Lan, "Real Time Non-intrusive Monitoring and Prediction of Driver Fatigue", IEEE Transport Vehicle Technology, July 2004.

[7] P. Smith, M. Shah and N. Da Vitoria Lobo, "Determining Driver Visual Attention With One Camera", IEEE Transactions on intelligent transportation systems, vol. 4, no.4, 2003.

[8] D. de Waard, The measurement of drivers' mental workload, ISBN 906807-308-7 Traffic Research Centre, University of Groningen, 1996.

[9] B.J. Wilson \& T.D. Bracewell "Alertness monitor using neural networks for EEG analysis", Neural Networks for Signal Processing X, vol. 2, 2000, pp. 814-820.

[10] A. Subasi, "Automatic recognition of alertness from EEG by using neural networks and wavelet coefficients", Expert systems with applications, vol. 28, 2005, pp. 701-711.

[11] S. Makeig, A.J. Bell, T-P. Jung and T.J. Sejnowski, "Independent Component Analysis of Electroencephalographic Data", Advances in Neural Information Processing Systems, vol. 8, MIT Press, Cambridge MA, 1996, pp. 145-151.

[12] C-T. Lin, R-C. Wu, S-F. Liang, Y-J. Chen and T-P. Jung, "EEG-based drowsiness estimation for safety driving using independent component analysis", Circuits and Systems I: Regular Papers, IEEE Transactions on [Circuits and Systems I: Fundamental Theory and Applications, IEEE Transactions on], vol. 52, no. 12, Dec. 2005, pp. 2726-2738.

[13] P.D. Welch, "The Use of Fast Fourier Transform for the Estimation of Power Spectra: A Method Based on Time Averaging Over Short, Modified Periodograms", IEEE Trans. Audio \& Electroacoust. , vol. AU-15, 1967, pp. 70-73.

[14] J. Ragot, M. Darouach, D. Maquin and G. Bloch, Validation de données par équilibrage de bilan Traité des nouvelles technologies, Série diagnostic et maintenance, Hermès, 1990, pp. 108-112.

[15] J.A. Hanley \& B.J. McNeil, "The meaning and use of the area under a receiver operating characteristic (ROC) curve", Radiology, vol. 143, no. 1, 1982, pp. 29-36.

[16] K. Karrer, T. Vohringer-Kuhnt, T. Baumgarten and S. Briest, "The role of individual differences in driver fatigue prediction", in Third International Conference on Traffic and Transportation Psychology, Nottingham, 2004. 